\title{
Sugar palm: challenges and opportunities
}

\begin{abstract}
Scientific studies on sugar palm fibers date from 2004. Before that, not much information can be found on the use of the fibers in engineering applications. The sugar palm is a multipurpose tree, and each part of the tree is used in daily life especially in rural areas. Research on the use of the sugar palm fibers in composite reinforcement started in 2005, and the topic has gained much attention ever since. There are both challenges and opportunities associated with the use of this natural resource, which are highlighted in this chapter.
\end{abstract}

